\title{
STRONGLY NORMAL CONES AND THE MIDPOINT LOCALLY UNIFORM ROTUNDITY
}

\author{
K. V. STOROZHUK
}

\begin{abstract}
We give the method of construction of normal but not strongly normal positive cones.
\end{abstract}

Keywords: normal ordered cone, extreme points, midpoint locally uniform rotundity.

\section{Cones, generated By a COnvex set on A Hyperplane}

Let $X$ be a normed ordered space and let $K=X_{+}$be its positive cone, which orders the space $X$ in the following way: $x \leq y \Leftrightarrow y-x \in K$. The cone generates $X$ if $X=K-K$. An (order) interval (or conic segment) $\langle a, b\rangle$ is the set $a+K \cap b-K$.

Let $a \in X$ and $B \subset X$. The number $\operatorname{dist}(a, B)=\inf \{\rho(a, b) \mid b \in B\}$ is called the distance between $a$ and $B$. The number $\widetilde{\operatorname{dist}}(A, B)=\sup _{a \in A} \operatorname{dist}(a, B)$ is called the nonsymmetric distance from the set $A$ to the set $B$. The distance between $A$ and $B$ is defined to be the number $\operatorname{dist}(A, B)=\min \{\widetilde{\operatorname{dist}}(A, B), \widetilde{\operatorname{dist}}(B, A)\}$.

Krein [1] introduced the notion of a normal cone. In our terminology, a cone is normal if the function $\rho(x, y)=\operatorname{dist}(\langle 0, x\rangle,\langle 0, y\rangle)$, defined on the set $K \times K$, is continuous at $(0,0)$.

In [2], there appeared the strong normality condition: it means the continuity of the function $\rho$ on the entire $K \times K$. It was also indicated there that it was not known whether every normal cone is strongly normal. See also [3] where the strong normality condition is actively used in Chapter 2.1 .

It is easy to see that the function $\rho$ is continuous on $K \times K$ if it is continuous at the points of the form $(x, x)$ with respect to one argument, i.e., when for any $x \in K$ $\rho\left(x, y_{n}\right) \rightarrow 0$ for $y_{n} \rightarrow x$. In what follows, the phrase like "function $\rho$ is continuous at the point $x$ " will mean exactly that.

It is also easy to see that, in checking continuity of the function $\rho$, it suffices to restrict ourselves to the analysis of its continuity at the points of a hyperplane section $\hat{B}$, i.e., to consider the sequences of the form $\hat{y}_{n} \in \hat{B}, \hat{y}_{n} \rightarrow \hat{x}$.

In the language of multivalued maps, strong normality is the continuity of the map $z \mapsto\langle 0, z\rangle$ at all the points $z \in K$. One can define a condition of "semi-strong" normality, which involves semicontinuity of the function $z \mapsto\langle 0, z\rangle$. Semicontinuity from above means that for $\hat{y}_{n} \rightarrow \hat{x} \widetilde{\operatorname{dist}}\left(\left\langle 0, \hat{y}_{n}\right\rangle,\langle 0, \hat{x}\rangle\right) \rightarrow 0$, whereas semicontinuity from below, that $\widetilde{\operatorname{dist}}\left(\langle 0, \hat{x}\rangle,\left\langle 0, \hat{y}_{n}\right\rangle\right) \rightarrow 0$.

In the present paper we give some examples of non-strongly normal cones. Our cones will be of the form $K=\{t \hat{x} \mid \hat{x} \in \hat{B}, t \geq 0\}$, where $\hat{B}$ is a convex set in the hyperplane section of $K$. These are good cones. We characterize strong normality of the cone $K$ in terms of geometry of the generating section $\hat{B}$.

Throughout this paper, $X$ is a real Banach space, $B \subset X$ is a bounded convex closed set in $X, E=\mathbb{R} \times X, K \subset \mathbb{E}$ is the cone generated by the set $\hat{B}:=\{1\} \times B$. 
It is clear that such cone is normal and if its interior is non-empty then $K$ generates the space $E$. We define a norm in $E$ by: $\|(t \times x)\|=|t|+\|x\|$. If $x \in X$ then by $\hat{x}$ we will denote the vector $(1 \times x) \in E$.

A segment $[a, b]$ in a vector space will be understood as the ordinary algebraic segment $\{a+t(b-a) \mid 0 \leq t \leq 1\}$. In particular, $[0, b]=\{t b \mid t \in[0,1]\}$.

Clearly, if $z \in K$ then $[0, z] \subset\langle 0, z\rangle$. By the thickness of the interval $\langle 0, z\rangle$ we will mean the distance from $\langle 0, z\rangle$ to the segment $[0, z] \subset\langle 0, z\rangle$.

It is not difficult to observe that $x$ is an extreme point of $B$ if and only if the thickness of the interval $\langle 0, \hat{x}\rangle$ equals zero, i.e., $[0, \hat{x}]=\langle 0, \hat{x}\rangle$ (see, for example, 4, Definition 1. 42 and Lemma 1. 43.]).

Theorem 1. Let $x \in B$. If the point $x$ is not extreme but there exist extreme points $y_{n} \rightarrow x$ then the function $\rho$ is discontinuous at $\hat{x}$.

Proof. The intervals $\left\langle 0, \hat{y}_{n}\right\rangle$ coincide with the segments $\left[0, \hat{y}_{n}\right]$ and converge in the limit to the segment $[0, \hat{x}]$, while the interval $\langle 0, \hat{x}\rangle$ is "thick" , i.e., it contains, besides the segment $[0, \hat{x}]$, some extraneous points. The rest is obvious.

It is already in $\mathbb{R}^{3}$ where there exists a convex closed set $B$ whose extreme points do not form a closed set. The reader can construct such an example him/herself or find an example, say, in 5, Chapter 4, the example after Corollary 18.5.3.

Corollary. In $\mathbb{R}^{4}$ there exists a normal but not strongly normal cone.

It is intuitively clear that all the unexpected «metamorphoses» of the intervals must take place on the boundary of the cone; the following theorem confirms this. The proof is not hard, but rather long. It is given at the end of the article.

Theorem 2. Let $B$ be a bounded convex subset of $X$. The function $\rho(x, y)$ is continuous at the interior points of the cone $K \subset E$.

Lemma on an extraneous point. Let $x \in B$ and $a \in X$.

1. The following conditions are equivalent:

(a) $[x-a, x+a] \subset B$,

(b) $\frac{\hat{x}+a}{2} \in\langle 0, \hat{x}\rangle \subset E$.

2. If $l(x)$ is the supremum of the lengths of the segments $B$ with the midpoint at $x \in B$ then the thickness of the interval $\langle 0, \hat{x}\rangle$ is no less than $\frac{\|l\|}{4}$ and no greater than $\|l\|$.

The proofs are rather simple and omitted here; we only present two illustrations.
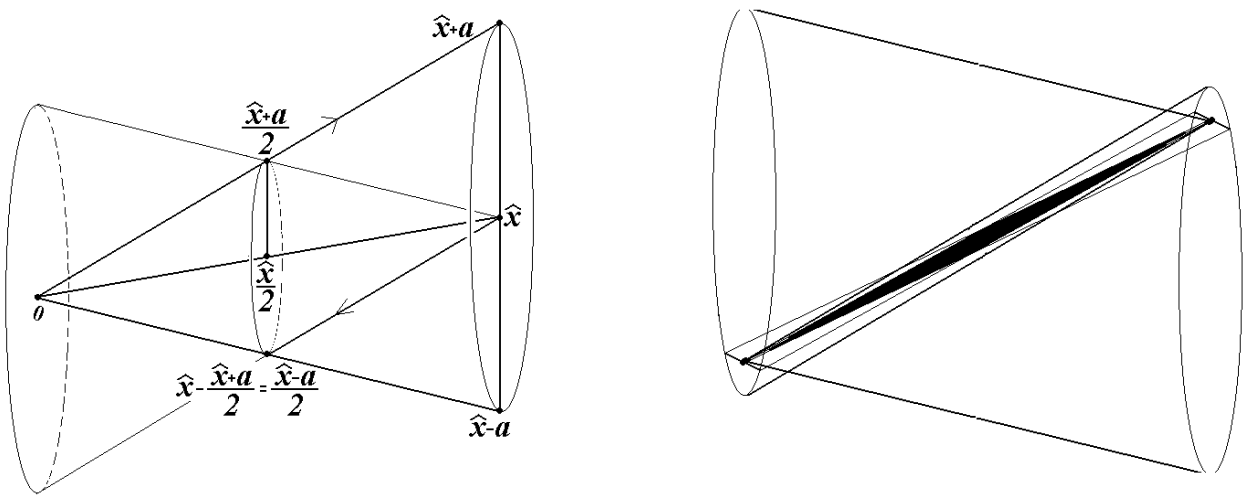
Lemma on the midpoints of long chords. Let $x \in B$ be an extreme point of the set $B$. The following are equivalent:

(a) there exists a sequence converging to $x$ and consisting of "uniformly nonextreme" points $y_{n} \in B$, i.e., the midpoints of the chords $B$ of the lengths greater than some $l>0$;

(b) the function $\rho$ is discontinuous at $\hat{x}$.

Proof. Since $x$ is an extreme point of $B$, we have $\langle 0, \hat{x}\rangle=[0, x]$.

Let $\hat{y}_{n} \rightarrow \hat{x}$. The segments $\left[0, \hat{y}_{n}\right]$ converge to the segment $[0, \hat{x}]=\langle 0, \hat{x}\rangle$. Therefore, the distance from the intervals $\left\langle 0, \hat{y}_{n}\right\rangle$ to $\langle 0, \hat{x}\rangle=[0, \hat{x}]$ converges to zero if and only if the thickness of the intervals $\left\langle 0, \hat{y}_{n}\right\rangle$ tends to zero. It remains to recall the second part of Lemma on an extraneous point. The lemma is proved.

Theorem on semicontinuity.

1. If $\operatorname{dim} X<\infty$, then the function $\rho$ is semicontinuous from below regardless of the set B generating the cone; i.e., the limit interval can only "grow up".

2. If $X$ is strongly convex, i.e., all the points of the unit sphere $S$ are extreme points of the ball $B$, then the function $\rho$ is semicontinuous from above, i.e., the limit interval can only decrease.

The first part of the theorem can be derived from the compactness of the finitedimensional ball and the reasoning that the reader carried out while proving the Lemma on an extraneous point. The second part follows from the fact that the intervals $\langle 0, \hat{x}\rangle$ coincide with the segments $[0, \hat{x}]$ for $x \in S$ and so on; cf. with the proof of Lemma on the long chords.

\section{Strong NORMALITY AND THE MidPoINT LOCALLY UNIFORM ROTUNDITY}

A space is called uniformly convex if $x_{n} \in S, y_{n} \in S$ and $\left\|\frac{x_{n}+y_{n}}{2}\right\| \rightarrow 1$ imply $\left\|x_{n}-y_{n}\right\| \rightarrow 0$. A space is called locally uniformly convex [6] if, in the previous definition, we additionally "fix" one of the endpoints of the chords: $x_{n} \equiv x \in S$.

If, on the other hand, we fix not an endpoint of the chords but their midpoints then we arrive at what K.W. Anderson 7 called the midpoint locally uniform rotundity (MLUR). (Anderson or somebody else attributed this property to G. Lumer and M. M. Day).

Namely, $X$ is midpoint locally uniformly rotund (MLUR) if whenever $x_{n} \rightarrow x \in$ $S,\left\|x \pm v_{n}\right\| \rightarrow 1$, we have $v_{n} \rightarrow 0$. It means geometrically that any point $x$ of the sphere $S$ is uniformly far from the midpoints of long chords of this sphere. It is precisely this property that is used in Lemma on the midpoints of long chords. It follows from this Lemma that, at the extreme point $x$ of the ball, the MLUR condition is equivalent to the continuity of the function $\rho$ at the point $\hat{x}$. Since at the interior points of the cone the function $\rho$ is always continuous (Theorem 2), the following theorem holds:

Theorem 4. Let $X$ be strongly convex and let $B$ be the unit ball in $X$. A cone $K \subset E$ is strongly normal if and only if $X \in M L U R$.

A substantial number of papers are devoted to the research on the MLUR property, its comparing with other characteristics of convexity of the sphere, the duality issues and the possibilities of the MLUR and non-MLUR renormalization. It is known that any separable Banach space is isomorphic to a locally uniformly convex and, therefore, MLUR space. On the other hand, the majority of "decent" spaces admit a non-MLUR renormalization. For example, the separable spaces, containing $l_{1}$, possess a strongly convex not-MLUR norm. See, [8, 9]. Let us refer, for 
example, to the paper [10; there one can find three examples of non-MLUR spaces. Let us give our own example for the sake of completeness of the exposition.

Example. A convex closed subset $B$ in a Hilbert space $H$ with an extreme point which is approximated by the centers of the infinite dimensional subdiscs.

Let $e_{i}, i=1,2, \ldots$ be a Hilbert basis in $H$. Let $B_{n}$ be the unit ball in the subspace spanned by the $e_{n+1}, e_{n+2}, \ldots$. For $n \geq 1$ we set $Z_{n}=\left\{t e_{1}+B_{n}|| t \mid \leq \frac{n}{n+1}\right\}$. Let $B$ be the closure of the convex hull of all $Z_{n}$. The interior of $B$ is non-empty (since the interior of $Z_{1}$ is non-empty: it contains a ball in $H$ of the radius $\frac{1}{2}$ ). The sequence $y_{n}=\frac{n}{n+1} e_{1}$ of the centers of the (infinite dimensional!) discs $y_{n}+B_{n} \subset B$ converges to the exterior point $e_{1}$ of the set $B$. Being considered as the unit ball, the set $B$ defines in $H$ an equivalent non-MLUR norm.

\section{Appendix. Proof of Theorem 2}

Let us note that if $K_{1} \subset K_{2}$ are two cones then for all $x\langle 0, x\rangle_{K_{1}} \subset\langle 0, x\rangle_{K_{2}}$. Secondly, if $K^{\prime}=T(K)$ where $T$ is a linear transformation then $T\langle 0, x\rangle_{K}=\langle 0, T x\rangle_{K^{\prime}}$.

Now, let $\hat{x} \in \operatorname{int}(K)$ and $\hat{y}_{n} \rightarrow \hat{x}$. Without any loss of generality, we can take $\hat{x}=(1 \times 0) \in \hat{B}$ (however, we keep in mind that $B$ is not necessarily a ball). We are going to prove that $\left\langle 0, \hat{y}_{n}\right\rangle \rightarrow\langle 0, \hat{x}\rangle$.

Let $\varepsilon>0$. Consider linear transformations $T_{+\varepsilon}$ and $T_{-\varepsilon}$ of the space $\mathbb{R} \times X$ which preserve the first coordinate and stretch the "second coordinate", i.e., the hyperplane $X$, by $1 \pm \varepsilon$ times: $f_{ \pm \varepsilon}(t \times z)=(t \times(1 \pm \varepsilon) z)$. The cone $K$ turns out to be in between the cones $f_{ \pm \varepsilon}(K)$.

The sets $B_{ \pm}=(1 \pm \varepsilon) B$ are separated from the set $B \subset X$; moreover, the distance from the boundaries of the sets $B_{ \pm}$to the boundary of the set $B$ is no less than the number $\varepsilon \cdot \delta$, where $\delta$ is the radius of the ball with the center at the point $x=0$, which is contained in $B$. Let us show this in the picture.

Let $l$ be a supporting plane at the point $D \in$ $B, D^{+}=(1+\varepsilon) D$. Clearly, the distance from $D^{+}$to the set $B$ is no less than $\left|C D^{+}\right|$. It follows from the similarity of the triangles $D A O$ and $D C D^{+}$that $\frac{C D^{\prime}}{O A}=\frac{D D^{+}}{O D}=\varepsilon$, therefore, $\left|C D^{+}\right|=\varepsilon|O A| \geq \varepsilon \delta$. A similar argument shows that the distance from $D$ to $B_{-}$is also is at least $\varepsilon \delta$. Thus, the set $B$ is included in the interval $B_{-} \subset B \subset B_{+}$ together with its $\varepsilon \delta$-neighborhood.

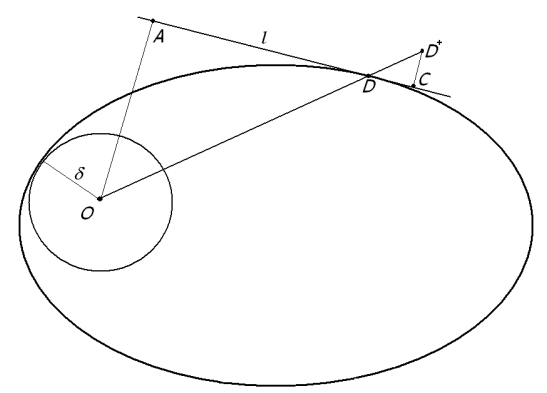

Let us consider further the linear transformations $G_{n}: \mathbb{R} \times X \rightarrow \mathbb{R} \times X$ which also preserve the first coordinate and move affinely the hyperplane $\hat{X}$ in such a way that the point $\hat{y}_{n}$ goes to 0 . Formally, $G_{n}(t \times z)=\left(t \times\left(z-t y_{n}\right)\right)$. Denote order intervals defined by the cones $G_{n}(K)$ by the symbol $\langle,\rangle_{n}$; whereas, the ones defined by the cones $f_{ \pm \varepsilon}(K)$, by the symbols $\langle,\rangle_{ \pm \varepsilon}$. It follows from the previous argument that if $\left\|y_{n}-x\right\|<\varepsilon \delta$, then $K_{-\varepsilon} \subset K_{n} \subset K_{+\varepsilon}$. Therefore, for the intervals we have

$$
T_{-\varepsilon}(\langle 0, x\rangle)=\langle 0, x\rangle_{-\varepsilon} \subset\langle 0, x\rangle_{n} \subset\langle 0, x\rangle_{+\varepsilon}=T_{+\varepsilon}(\langle 0, x\rangle) .
$$

Recall now that $\langle 0, x\rangle_{n}=G_{n}\left\langle 0, G_{n}^{-1} x\right\rangle=G_{n}\left\langle 0, y_{n}\right\rangle$. However, for large $n$ the intervals $G_{n}\left(\left\langle 0, y_{n}\right\rangle\right)$ are close to the intervals $\left\langle 0, y_{n}\right\rangle$. Thus, for large $n T_{-\varepsilon}(\langle 0, x\rangle) \subset$ $\left\langle 0, y_{n}\right\rangle \subset T_{+\varepsilon}(\langle 0, x\rangle)$. Since, for small $\varepsilon$, the intervals $f_{ \pm}\langle 0, x\rangle$ are close to the interval $\langle 0, x\rangle$, the theorem is proved. 


\section{REFERENCES}

[1] Krein, M. Proprie'te's fondamentales des ensembles coniques normaux dans l'espace de Banach. (French) C. R. (Doklady) Acad. Sci. URSS (N. S. ) 28, (1940). 13-17.

[2] Emelyanov, E. Yu. ; Wolff, M. P. H. Positive operators on Banach spaces ordered by strongly normal cones. Positivity 7 (2003), N. 1-2, 3-22.

[3] Emel'yanov. E.Yu. Non-spectral asymtotic analysis of one-parameter operator semigroups. Operator theory Advances and applications, vol.173. Birkhauser 2007.

[4] Aliprantis, Charalambos D. ; Tourky, Rabee. Cones and duality. Graduate Studies in Mathematics, 84. American Mathematical Society, Providence, RI, 2007.

[5] R.T. Rockafellar. Convex Analysis. Princeton University Press, Princeton,. N.J., 1970.

[6] A. R. Lovaglia. Locally uniformly convex Banach spaces, Trans. Amer. Math. Soc. 78, (1955), 225-238.

[7] K. W. Anderson. Midpoint local uniform convexity, and other geometric properties of Banach spaces, Ph. D. dissertation, Univ. Illinois, Urbana, IL, 1960.

[8] Kadec', M. Ǐ. Spaces isomorphic to a locally uniformly convex space. (Russian) Izv. Vysš. Učebn. Zaved. Matematika 19591959 no. 6 (13), 51-57.

[9] Kadec', M. İ. Relation between some properties of convexity of the unit ball of a Banach space. Funct. Anal. Appl. 16, N 3, 204-206.

[10] Smith, Mark A. Some examples concerning rotundity in Banach spaces. Math. Ann. 233 (1978), no. 2, 155-161.

Konstantin Storozhuk

Sobolev Institute of Mathematics,

acad Koptyug avenue, 4,

NOVOSIBIRSK STATE UNIVERSITY,

PIROGOVA STR, 2,

630090, Novosibirsk, Russia

E-mail address: stork@math. nsc. ru 